\title{
The quality of service of the deployed LTE technology by mobile network operators in Abuja-Nigeria
}

\author{
Anabi Hilary Kelechi ${ }^{1}$, Uyi Aiyudubie Samson ${ }^{2}$, Matthew Simeon ${ }^{3}$, Okoyeigbo Obinna ${ }^{4}$, \\ Aligbe Alex ${ }^{5}$, Atayero Aaron Aderemi ${ }^{6}$ \\ ${ }^{1,2,4,5,6}$ Department of Electrical and Information Engineering, Covenant University, Ota, Nigeria \\ ${ }^{3}$ Department of Electrical and Electronics Engineering, Federal University of Agriculture, Abeokuta, Nigeria
}

\begin{tabular}{l} 
Article Info \\
\hline Article history: \\
Received May 13, 2020 \\
Revised Jan 6, 2021 \\
Accepted Oct 17, 2021 \\
\hline
\end{tabular}

\section{Keywords:}

Downlink throughput

LTE technology

QoS

RRC connection drop rate

Uplink throughput

\begin{abstract}
In this study, the real-world performance analysis of four Nigerian mobile network operators (MNOs), namely MTN, GLO, Airtel, and 9Mobile longterm evolution (LTE) cellular network, were analyzed and compared. The Nigerian MNOs utilize $5 \mathrm{MHz}, 10 \mathrm{MH}$, and $20 \mathrm{MHz}$ channel bandwidths based on third-generation partnership project's (3 GPPs) recommendation. The presented analysis shows the uplink (UL), and downlink (DL) throughputs gaps in mobility condition as well as other LTE's system quality of service (QoS) key performance indicators (KPI's) of: Connection drop rate, connection failure rate, peak physical downlink throughput, minimum radio link control (RLC) downlink throughput threshold and latency are not strictly followed. The reason may be due to a lack of regulatory oversight enforcement. The comparative studies showed that MTN provides the best QoS. The introduction of novel LTE QoS metrics herein referred to as national independent wireless broadband quality reporting (NIWBQR) is the significant contribution of this study. The goal of this study is to show the quality of the network as it affects the user's experience. Important observation showed that all the MNOs are not adhering to the 3 GPPs specified user plane latency of $30 \mathrm{~ms}$ and control plane latency of $100 \mathrm{~ms}$, respectively, which makes video streaming and low latency communication a near-impossible task.
\end{abstract}

This is an open access article under the CC BY-SA license.

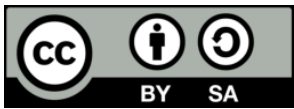

\section{Corresponding Author:}

Uyi Aiyudubie Samson

Department of Electrical and Information Engineering

Covenant University

Ota, Nigeria

Email: aiyudubie.uyi@covenantuniversity.edu.ng

\section{INTRODUCTION}

The Nigerian mobile data market has witnessed a broadband revolution in terms of the readily available number of laptop computers, tablets, notebook, and smartphones accessing wireless connectivity. Furthermore, there is a paradigm shift on users' demand for applications and services from mobile communication systems, which go far beyond just voice telephony. According to the Nigerian communications commission (NCC) [1], the total of all active telephony subscribers in Nigeria increased from 142,320,120 in Nov 2018 to $169,104,830$ in Nov 2018. This is equivalent to a $19.30 \%$ change in teledensity. It is of official notice that the Nigerian mobile data market is made up of Internet-savvy consumers whose consumption of mobile apps for social, corporate, video, and music streaming are on the increase $[2,3]$. Long term evolution (LTE) and advanced long term evolution (advanced-LTE) technology are already consolidated technologies reaching the maturity stage and expected to be fully deployed by mobile 
vendors. These two technologies have become the mainstream radio access network as the previous wireless generations of $2 \mathrm{G}, 3 \mathrm{G}$ is insufficient to handle such a vast subscriber base $[4,5]$. In other to meet with these challenges (LTE)/(LTE-Advanced), are extensively deployed in some notable Nigeria towns release 8 and 9 [6].

The LTE market in Nigeria is dominated by the operators listed in Table 1. As could be seen from Table 1, the LTE operators operate both unpaired spectrum time division duplex (TDD) mode and paired frequency division duplex (FDD) mode over a dynamic flexible channel bandwidth of 5, 10, 15, $20 \mathrm{MHz}$ respectively. All these dynamic flexible channel bandwidths are allocation in the UHF and the ISM band. Ordinarily, LTE is an all IP network that targets mobile data delivery and can support voice services using voice over LTE (VoLTE) infrastructure. The technology admits the support for bandwidth sensitive applications like video-on-demand services as the Nigerian market is tending towards massive mobile data consumption [7]. Over-the-top (OTT) applications comprising of television (e.g., Apple TV, Youtube), voice calling (e.g., Skype, Zoom), message (e.g., WeChat, WhatsApp) should run smoothly on the LTE infrastructures. The OTT is a concept in which the content providers bypass the telecommunication providers and sell their audio, video, and web content directly to the consumer. This implies that the deployed LTE infrastructure should be able to meet the IMT-advanced advocated peak data rate of $1 \mathrm{Gbps}$ and $100 \mathrm{Mbps}$ for low and high mobility specifications, respectively.

Table 1. Nigerian LTE service providers [8]

\begin{tabular}{ccccc}
\hline Service Provider & Spectrum Allocation & Channel Bandwidth $(\mathrm{MHz})$ & Duplex mode & Band No \\
\hline 9Mobile & 1872.6 & $5 \mathrm{MHz}$ & FDD & 3 \\
MTN & 2660 & 20,5 & FDD & 7,20 \\
Bitflux (E-stream) & 2370,2385 & 20,10 & TDD & 40 \\
GLO & 783 & 10 & FDD & 28 \\
Airtel & 1857 & 5 & FDD & 3 \\
Ntel & $942.5,1812.5$ & 5,15 & FDD & 8,3 \\
Spectranet & 2350 & 20 & TDD & 40 \\
Smile & 816 & 10 & FDD & 20 \\
Swift & 2330 & 20 & TDD & 40 \\
\hline
\end{tabular}

Furthermore, end-to-end (E-2-E) QoS, voice over internet protocol (VoIP), backhaul efficiency, and latency should be lower than voice traffic over UMTS circuit-switched networks. The optimal LTE user plane latency is within the threshold of $30 \mathrm{~ms}$. Based on Rel. 8 specification, which is the first deployed LTE standard, LTE peak downlink rate is 326.4 Mbps using $4 \times 4$ antenna configuration and 172.8 Mbps for $2 \times 2$ antenna architecture while deploying $20 \mathrm{MHz}$ channel bandwidth. At the same time, the expected peak data rate using the $20 \mathrm{MHz}$ spectrum channel bandwidth is about 50 and $100 \mathrm{Mbps}$ downlink and uplink, respectively assuming two receive antenna and two transmit antennas at the terminals. There is a diversity gain in $4 \times 4$ antenna configuration at the expense of microprocessor complexity and energy consumption. In a market with a weak institutional framework, how well the mobile network operators (MNOs) meet the above LTE specifications remains a critical question. Therefore, there is a need for an independent body to investigate the deployed QoS of MNOs. The problem addressed in this paper is: Is the deployed LTE network in Nigeria capable of sustaining the user quality of experience (QoE). Hence, our main contributions are;

- Investigate the QoS on some of the deployed commercial LTE networks in Nigeria utilizing via drive test.

- Compare the QoS of some of the Nigerian LTE MNOs using some of the standard key performance indicator (KPI) metrics.

- Design national independent wireless broadband quality reporting (NIWBQR) service.

- Provide an LTE QoS dataset for future work and analysis on demand.

The rest of this paper is organized as follows, section 2 focuses on the related works. Section 3 gives an overview of LTE technology. Section 4 discusses some of the key LTE QoS metrics and test environment. Section 5 discusses the result while section 6 presents the conclusion.

\section{RELATED STUDIES}

Prior efforts have been made to analyze the QoS of LTE network, both from the theoretical perspective and the field trial experiments [9-17]. LTE QoS simulation analysis, focusing on the LTE spectrum assigned to the Malaysian operators, which is $2.6 \mathrm{GHz}$ was performed by [9]. Before the study, the Malaysian LTE consumers were to anticipate up to $37.7 \mathrm{Mbps}$ at the uplink and $43.2 \mathrm{Mbps}$ IP layer throughput at the downlink using SISO or MISO configuration. An in-depth study of application behavior on 
performance and the effect of network protocol both in a controlled environment and commercially deployed LTE network was done by $[10,11]$. Different network statistics involving the TCP and UDP protocols were analyzed before this study. The power and performance characteristics of 4G LTE networks was investigated by [12], the study observed that LTE achieved higher uplink and downlink throughput compared to UMTS and wireless fidelity (WiFi), having a median value of $6 \mathrm{Mbps}$ and $13 \mathrm{Mbps}$, respectively. To improve the speed of analyzing the LTE radio access networks (RAN), [12] proposed CellScope. CellScope is a system designed to apply a domain-specific application and formulation of multi-task learning (MTL) to RAN performance analysis. Some of the factors that could lead to dropped in QoS of LTE RAN has been identified as user mobility, inefficient algorithms, skewed traffic pattern, interference, lack of coverage, equipment failure, software bugs, unoptimized configuration parameters and protocol errors [13]. The performance problems of a network can be easily discovered by monitoring aggregate metrics, which includes; rate throughput per cell in-time scales covering long time windows and connection drop. Obviously, the degradation metrics is performed using error-prone analysis, manual, and time-consuming [14, 15]. In recent times, drones have become an integral part of the wireless ecosystem, and LTE technology, especially Release 15, has shown tremendous support for providing the radio connectivity [16, 17].

In a similar experiment, the use of the LTE backbone was investigated to provide a wireless connection for an unmanned aerial vehicle (UAV) [18]. In the research, some of the LTE QoS metrics, such as reference signal received quality (RSRQ) and reference signal received power (RSRP) and were analyzed. The results from the experiments indicate that using the $50^{\text {th }}$ percentile RSRPs at the heights of $50 \mathrm{~m}$ and $150 \mathrm{~m}$, the received signal strengths are $18.9 \mathrm{~dB}$ and $15.6 \mathrm{~dB}$ higher than the corresponding value at ground level, respectively. From the plethora of studies analyzed, none of the studies were able to propose a collection of metrics to grade the offered LTE user QoS. Hence, this forms the basis of this study.

\section{OVERVIEW OF LTE SYSTEM ARCHITECTURE}

LTE has evolved from release 8 to release 9, release 10, and beyond all, maintaining the same system architecture though with slight improvement. Release 8 laid the foundation by introducing some notable technologies previously not utilized in the older wireless generation. Some of these technologies include; Orthogonal frequency-division multiple access and single-carrier frequency-division multiple access on the downlink and uplink, respectively [19]. The two schemes are motivated by orthogonal frequencydivision multiplex (OFDM), which is an offshoot of a multiplexing scheme that is frequency division multiplexing (FDM). The OFDM concepts are based on the principle of splitting the wideband into narrowbands, often referred to as subcarriers or frequency bins. The spacing of frequency between the carriers provides orthogonality among subcarriers, which subsequently eliminates the subcarrier interference and reduces inter-symbol interference (ISI) [20]. While the standard adopted $15 \mathrm{kHz}$ subcarrier spacing for normal applications, $7.5 \mathrm{kHz}$ was preferred for multicast broadcast single-frequency network (MBSFN) mode of the LTE. Therefore, there is no need for guard bands as required by FDM. The subcarrier architecture ensures that LTE performs robustly in multipath fading environment and deploying cyclic prefix (CP); as a result, the challenge of delay spread that causes ISI is eliminated. The length of CP utilized is environmentally dependent, as different environments such as urban and rural areas have different needs. LTE also supports single and multiple users-multiple input-output. Single User-multiple input multiple out (SU-MIMO) is a concept in which the eNodeB (eNB) takes advantage of diversity gains, which may be spatial, transmit and receiver beam signals to a user exploiting multipath signal component [21]. While in multiple user-multiple input multiple outputs (MU-MIMO), the eNB beams signals to numerous users simultaneously aiming to increase system capacity and spectral efficiency. Release 9 offered improved throughput by introducing the heterogeneous network (HetNet) concept. Originally, LTE is an IP system and to support voice communications, voice over LTE (VoLTE), and enhancements to circuit switch fallback (CSFB) to universal mobile telecommunications service (UMTS)/global system for mobile communications (GSM) was introduced by LTE release 9. This concept was initially introduced in release 8 as a temporary solution to support voice on LTE system. The LTE release 10 and beyond, often referred to as LTE/LTE-A introduced improve throughput and spectral efficiency by initiating carrier aggregation (CA). This is a concept of using non-contagious spectrum channels for transmission and higher-order MIMO. A typical LTE deployment scenario can be seen from Figure 1. From the radio frequency side (RF), the LTE consists of a remote radio head (RRH), which is in close proximity to the antenna. The RRH is connected to the baseband unit $(\mathrm{BBU})$ via fiber located in the cell cabinet. The same fiber links the eNBs to the evolved packet core (EPC)/core networks where packet processing takes place.

To address interference issues, release 10 introduced schemes such as enhanced interference cancellation, coordinated multipoint transmission (CoMP), which includes improved intercell interference coordination (eICIC), which are all advanced management schemes. These strategies enhance cell-edge 
throughput and capacity-the eNB incorporated user plane in addition to control plane configuration. The evolved UMTS terrestrial radio access (E-UTRA) layer, radio link control (RLC) layer, medium access control (MAC), packet data convergence protocol (PDCP) layer, physical (PHY) layer were introduced in the user plane. Also, the radio resource control (RRC) layer protocol is overseen by the control plane. The system architecture evolution (SAE) of LTE consists of eNB connected to the evolved packet core through the standard interface (S1). The eNBs are connected to one another using the interface of X2. The eNB uses the S1-MME to connect to the mobility management entity (MME) and uses S1-U to connect to the serving gateway (S-GW). LTE achieves control plane signaling, authentication, roaming, security control, and radio bearer management via the use of MME. The S-GW provides inter-radio access technology (inter-RAT) mobility, packet routing, and forwarding, and transport-level QoS mapping. Packet data network (PDN) gateway (P-GW) performs the following task of user equipment (UE) IP address allocation, policy enforcement, and packet filtering.

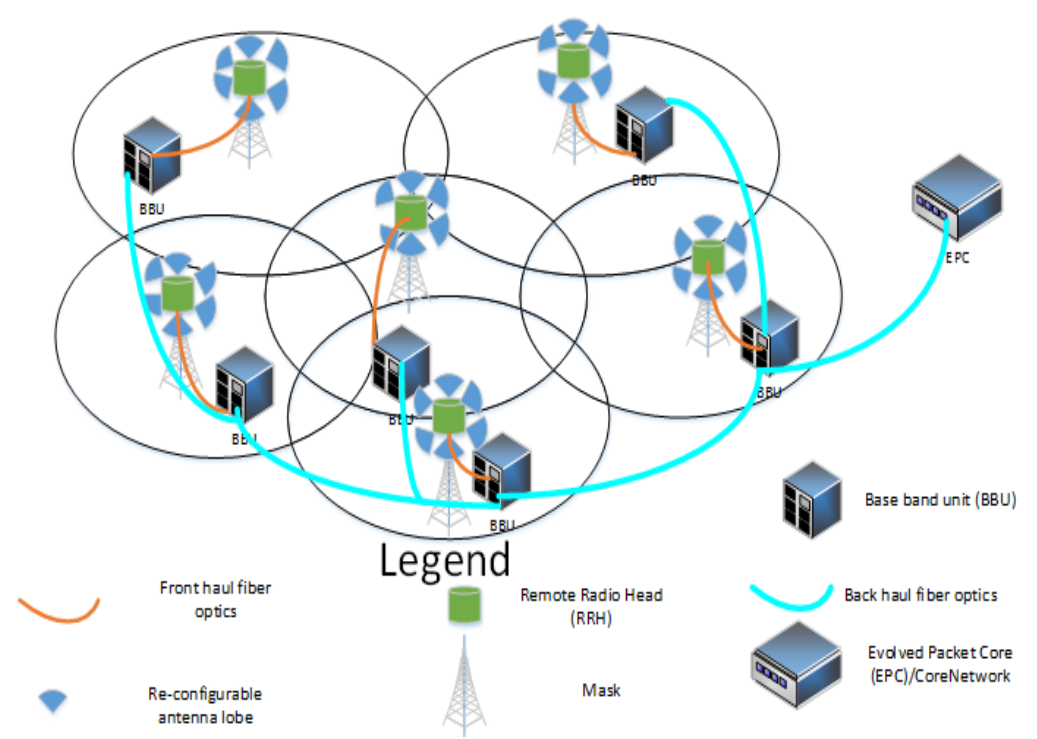

Figure 1. Schematic representation of LTE deployed scenario

\subsection{The Nigerian fibre technology}

The overall LTE performance depends on the state of the fiber backbone. The fiber technology was introduced in LTE standard to address the low latency and low capacity associated with using either microwave link or copper wire. Fiber can be deployed in three LTE frontiers: i) the access; ii) backbone/backhaul; and iii) the core [22]. The access connects the LTE RRH to the front haul BBU located in the site cabinet. The backbone connects the different base transmission stations (BTS). BTS might be in the same locality or across different localities. Furthermore, the connection between the BTS and mobile switching center (MSC) is implemented via the fiber backhaul. The core is responsible for linking the national wireless traffic to international networks. Overall, as of 2014, the core fiber has a distance of $16,930 \mathrm{~km}$, while the access and backhaul fiber distance is $64,433 \mathrm{~km}$ [23]. By implication, Nigeria has more core fiber cable length than the access and backhaul. It is believed that this figure has increased significantly over the years. The major players in the fiber are Airtel, MainOne, Globacom, MTN, and Dolphin. The installed fiber core carrying capacity as of 2014 is 27.62 Tbps. Interestingly, the majority of the fiber core providers are also providing LTE mobile data. Hence, issues arising from inter-operator interconnectivity is expected to be minimal. Theoretically, this will lead to seamless connectivity and low call-drops, inter BTS handover-failure, S1/X2 handover failure rate, and packet latency-round-trip delay.

\section{TEST PARAMETER DEFINITION}

3GPP-3rd generation partnershipproject, through the technical specifications (TS), has proposed several tests aimed towards enhancing the functionality of the eNB and UE. Broadly, these tests can be categorized as signal power, signal quality, spectrum, and intermodulation distortion. In this section, the LTE experimental environment is described. The various LTE QoS and the NIWBOR concept are also discussed. 


\subsection{Test environment}

The test environment consists of collocated eNBs of various LTE MNOs in Nigeria, supporting various channel bandwidth configuration of earlier stated above. The test settings include a UE jacket containing several Samsung galaxy LTE smartphones/UE capable of taking LTE measurement and tracing tool. The UE jacket supports the collection of the drive test result from different MNO simultaneously. The different UEs test results were logged to a laptop installed with rand advisor E6474A drive test software. The software has the capacity to undertake RF walk and mobility tests. The average speed for the mobility test was $50 \mathrm{~km} / \mathrm{h}$ and was performed at a onetime instance. Furthermore, the test routes are areas reckoned with high mobile traffic usage during the test period because of the presence of many commercial offices around. For LTE, the terminal supports $100 \mathrm{Mb} / \mathrm{s}$ on DL and $50 \mathrm{Mb} / \mathrm{s}$ on UL with $2 \times 2 \mathrm{MIMO}$ (multiple-input and multiple outputs) and 64 QAM on DL and 16 QAM on UL for modulation. About $35 \mathrm{~km}$ with an urban morphology, known as the mobility route is covered by the drive test route.

\subsection{RF test metrics}

The RF metrics are crucial to the overall of the deployed LTE networks as it displays the state of physical channel parameters. Several signals of interest can be analyzed in the LTE RF domain. In this study, we focus on the signal-to-noise ratio (SNR) defined by the 3GPP specification based on 2X2 MIMO system as [24]:

$$
S N R=\frac{\dot{E}_{S}^{(1)}+\dot{E}_{S}^{(2)}}{N_{o c}^{(1)}+N_{o c}^{(2)}}
$$

where $E_{S}^{(1)}$ denotes the received energy per resource element (RE) of the useful part excluding the cyclic prefix (CP) with the superscript (1) denoting the antenna number 1 in the $2 \times 2$ MIMO configuration. Since there are many REs in LTE resource block $(\mathrm{RB})$, the total power will be averaged across the RBs. $N_{o c}^{(1)}$ denotes the power spectral density (PSD) of a white-noise source with the superscript (1) representing the antenna number 1 in the 2x2 MIMO configurations. LTE, just like any other wireless, might experience interference as a result of co-channel or adjacent interference which is modeled as a signal-to-interferenceplus-noise ratio (SINR), mathematically defined as:

$$
S I N R=\frac{\hat{E}_{s}^{(1)}+\dot{E}_{s}^{(2)}}{N_{o c}^{(1)}+N_{o c}^{(2)}+1}
$$

the $I$ denotes the source of interference signals measured in $\mathrm{dB}$ or $\mathrm{dBm}$. For accurate symbol decoding, the SINR value has a direct implication on channel quality indicator (CQI). A good CQI value indicated that SINR must be higher than a specific BLER target, $\theta$ and mathematically stated as:

$$
C Q I=S I N R \geq \theta
$$

The reported CQI measurements set a benchmark that must be met before LTE transmission commences. Given that CQI meets the minimum requirement, it is possible to estimate the throughput as [25].

$$
\text { Throughput }=N_{\text {symb }}^{D L} N_{S C}^{R B} R E
$$

where $N_{\text {symb }}^{D L}$ connotes the number of modulated symbols in the downlink (DL) and the $N_{S C}^{R B}$ denotes the number of subcarriers per RB and RE indicates resource element.

\subsection{LTE protocol testing}

While RF test parameters measure the received power in $\mathrm{dB}$ and $\mathrm{dBm}$, to verify signaling functionality like state handling, call setup and release, mobility and call reconfigurations protocols are needed. The PHY layer, MAC, RLC, and PDCP are some of the protocols that interface between the eNB and the UE. The PHY handles the channel coding, modulation, scrambling, and OFDM RE mapping. The MAC layer performs the logical to physical channel mapping as well as negotiated QoS. The responsibility of retransmission requests in the MAC is carried out by the hybrid automatic repeat request (HARQ). The RLC performs packet data segmentation to reflect the state of the underlying physical channel. In a situation that a segment is lost during transmission, the RLC can evoke retransmission via the automatic repeat request (ARQ). The RLC ARQ and the MAC HARQ are entrusted with retransmission functionality. The PDCP consists of user data and control. The PDCP performs data ciphering and compression of the user data. 
Furthermore, during handover PDCP assist by performing lossless handover and in-sequence delivery. The PDCP achieves data integrity protection through the control data. The focus of this LTE performance analysis test was on the air interface protocol of the user plane. Driven by the need to investigate the performance of the different LTE layers, the total uplink (UL) and downlink (DL) throughputs were analyzed separately.

\subsection{National independent wireless broadband quality reporting (NIWBQR) QoS metrics}

Some of the issues addressed by LTE/LTE-A are as follows, multi-antenna support, higher radio access data rates in the range of Mbps, enhanced system capacity and coverage, adaptive bandwidth operations, enhanced spectral efficiency, low latency, reduced operating costs and seamless integration with the Internet and existing mobile communication systems. How well does deployed LTE meet the aims and objectives are the goal of national independent wireless broadband quality reporting (NIWBQR) Metrics. From Figure 2, it could be seen that NIWBOR metrics consist of RRC connection drop rate, RRC connection setup failure rate, latency, normalized packet DL throughput, non-LTE UE technology, fraction bins with throughput less than $500 \mathrm{kbps}$.

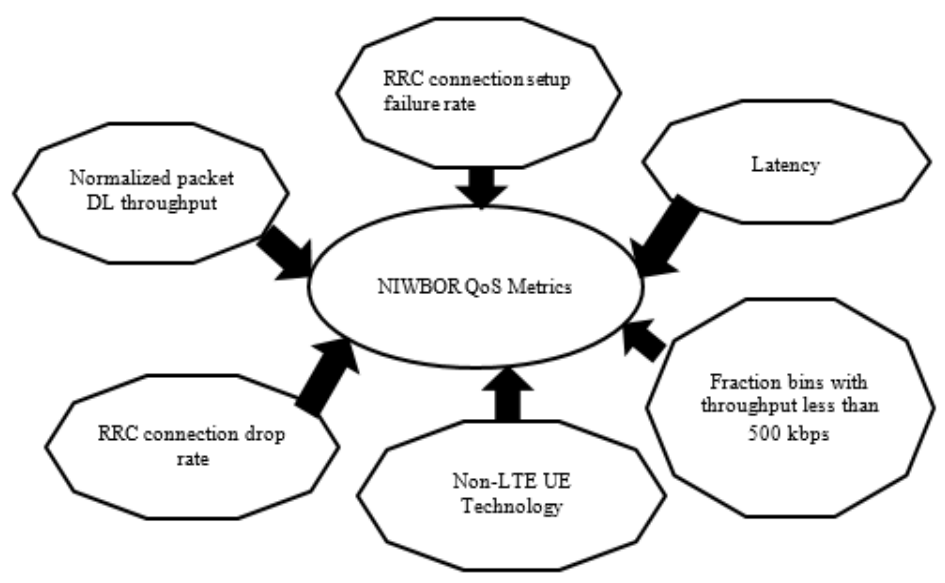

Figure 2. NIWBOR QoS metrics

NIWBQR metrics is thus defined as the weighted sum in which the core LTE QoS indicators of: non-LTE UE technology, RRC connection drop rate, RRC connection setup failure rate, normalized packet DL throughput, a fraction of bins with DL RLC layer throughput less than $500 \mathrm{kbps}$ and network latency deviates from the 3GPP laid operational standard. The NIWBOR rank of MNO operator $i$ mathematically defined as:

$$
\operatorname{NIWBOR} \operatorname{Rank}(i)=\sum_{1=i}^{n} w_{i}\left(\alpha_{i}+\beta_{i}+\gamma_{i}+\varphi_{i}+\emptyset_{i}+\vartheta_{i}\right)
$$

where $\sum_{1=i}^{n}$ denotes the sum of NIWBOR parameters, $w_{i}$ denotes the NIWBOR weighted score many parameters weighted parameters summed to 100 that determines the NIWBOR score, $\alpha_{i}$; denotes non-LTE UE technology, $\beta_{i}$; RRC connection drop rate, $\gamma_{i}$; RRC connection setup failure rate, $\varphi_{i}$; denotes normalized packet DL throughput (Mbps), $\varnothing_{i}$; denotes fraction of bins with DL RLC layer throughput less than $500 \mathrm{kbps}$ and $\vartheta_{i}$; denotes network latency (ms). Note that the thresholds are adjustable. For example, the minimum DL throughput can be changed from $500 \mathrm{Kbps}$ to $10 \mathrm{Mbps}$ if the development level within the country warrants this.

\section{RESULTS}

The performance analyses commercially deployed collocated 3GPP release 8 LTE networks by Four (4) MNO based single user- multiple inputs multiple outputs (SU-MIMO) were presented. Traditionally, the end-users throughputs are validated by conducting application layer data rate conformance tests comprising: TCP/IP, Youtube, and Facebook. The goal of this analysis is to focus on the physical layer of the LTE protocol stack, which also reflects the state of the higher protocol layer stacks. The dataset used in this work was performed in 2018 in the Abuja metropolis. The raw dataset is available on request at the parent company Enext wireless Inc. 


\subsection{Accessibility test}

Regardless of the LTE mode, either FDD or TDD, unicast, multicast, and broadcast, there are mandatory handshake setup protocols and signals that must be established for data to be transmitted between the UE, eNB and the EPC. Accessibility tests are, therefore, initialization procedures to secure the data transmission link between the UE, eNB, and the EPC. The LTE is an all data-driven RAN in which for a packet data to transmission from EPC to UE, the bearer, radio resource management (RRM), the control channels information must be secured. Some of these control channels are; common control channel $(\mathrm{CCCH})$, dedicated control channel $(\mathrm{DCCH})$, physical uplink control channel (PUCCH), broadcast control channel $(\mathrm{BCCH})$, paging control channel $(\mathrm{PCCH})$, and multicast control channel $(\mathrm{MCCH})$. Each of the channels performed specific functions towards the overall superior performance of LTE technology. The LTE performance accessibility metrics is a collection of initial LTE set up protocols such as; attach failure rate, service request failure rate, $\mathrm{RRC}$ setup failure rate, attach delay, and service request delay.

For the purpose of this study, we focus on service request delay. Service request delay is the delay encountered by the UE. In order to access the physical random access channel (PRACH), the UE sends its information request by keying into the $\mathrm{PCCH}$, which is mapped to the paging channel (PCH). The $\mathrm{PCCH}$ and $\mathrm{PCH}$ are responsible for granting access to the physical downlink control channel (PDCCH), which oversees the decoding of the downlink control information (DCI) signals. The 3GPP standard specifies the physical control format indicator channel (PCFICH) mandates the UE to communicate to the eNB to obtain the control format information (CFI), which contains the information necessary to decode PDCCH information. Apart from the RRC and RRM, UE must acquire the DCI for proper decoding of relevant information to be admitted to the network. Signaling is provided by the network within system information block (SIB) Type 2, and higher layers provide the PRACH configuration. This also includes the preamble initial target power (PITP). The PITP simply denotes the power level that the UE will send the preamble the first time to the network and how much the power level will be increased if the preamble is not acknowledged. The service request delay of the four (4) MNOs in Nigeria was examined, as showed in Figure 3. Clearly shows that MTN has the worst service request delay while AIRTEL has the best setup time. This implies that the MTN backbone cannot be adopted as the backhaul for emergence disaster communications and as well as disaster management due to its excessive setup time. Moreover, applications that request low service setup rate will experience weak performance degradation. Apparently, the MNO with the worst service request delay is not the ideal candidate for low latency communication gadgets and sensors. It can further be extended to imply that battery efficiency will be weak as more time is needed to secure the link, which invariably leads to longer battery consumption time.

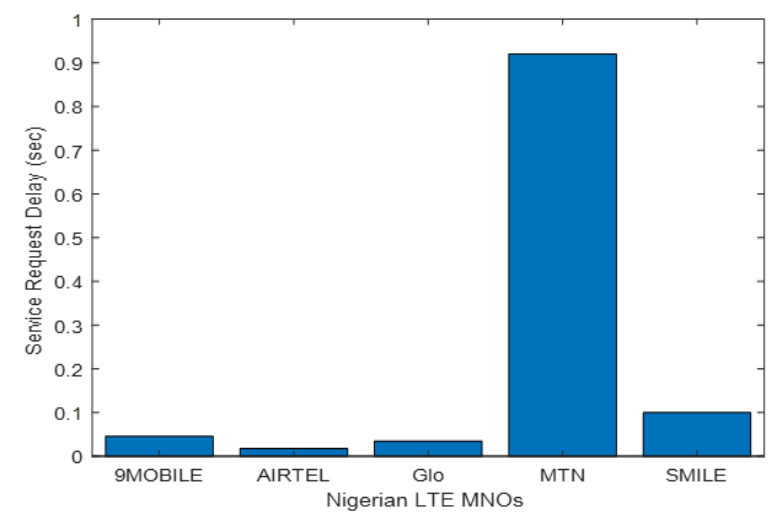

Figure 3. Comparison of the service request delay time among the Nigerian LTE MNOs

\subsection{Integrity test}

An integrity test is a cross-layer approach to analyzing the performance of the deployed LTE networks. The integrity test is a collection LTE metrics test comprising: Physical layer throughput DL, DL RLC throughput peak, UL RLC throughput peak, RLC ARQ/HARQ retransmission rate, packet latencyround-trip delay. Intuitively, the above test is categorized as the physical layer, the MAC layer, the radio link layer, and the TCP/IP layer. It is an exhaustive test analyzing the performance of the E-UTRAN user plane (PDPC/RLC/MAC/PHY) while ignoring the control plane (RRC).

The main requirements needed for this design of LTE system were identified during when the standardization work was carried out in 2004. The peak data rates targets were $100 \mathrm{Mbps}$ (downlink) and 
$50 \mathrm{Mbps}$ (uplink) for $20 \mathrm{MHz}$ spectrum allocation. How well does the deployed LTE technology by Nigerian MNO meet this specification is the focus of physical layer throughput DL, DL RLC throughput peak, UL RLC throughput peak. As could be seen from Figure 4, none of the Nigerian LTE MNOs were able to attain the targeted 100 Mbps (downlink). The best MNO was MTN, which achieved about $85 \mathrm{Mbps}$, and the worst was Glo, which achieved below $25 \mathrm{Mbps}$. When the median value was analyzed, 9MOBILE performed poorly. It can be deduced that the MIMO configuration might not be configured appropriately and needs to be re-examined. Figure 5 examined peak and median physical layer throughput UL among the Nigerian LTE MNOs.

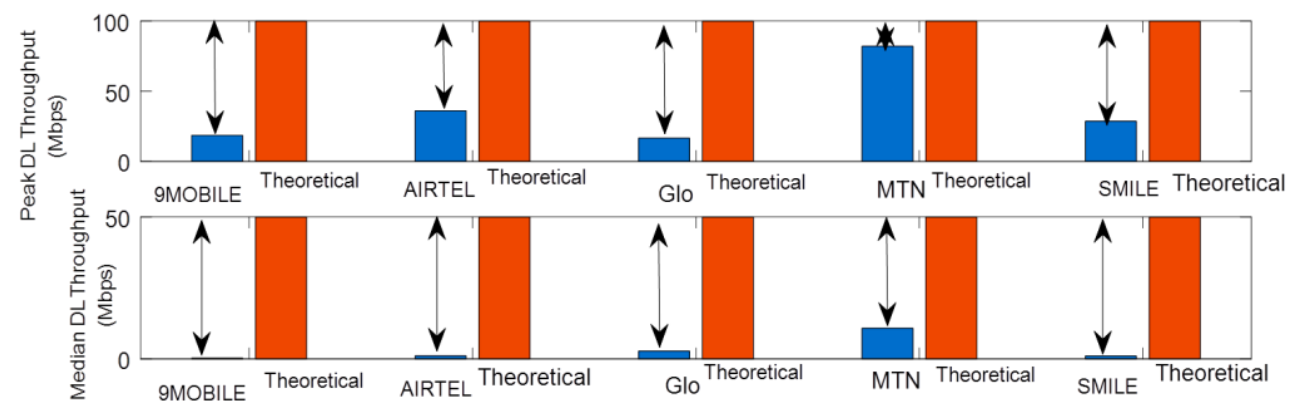

Figure 4. Peak and median physical layer DL throughput comparison among the Nigerian LTE MNOs

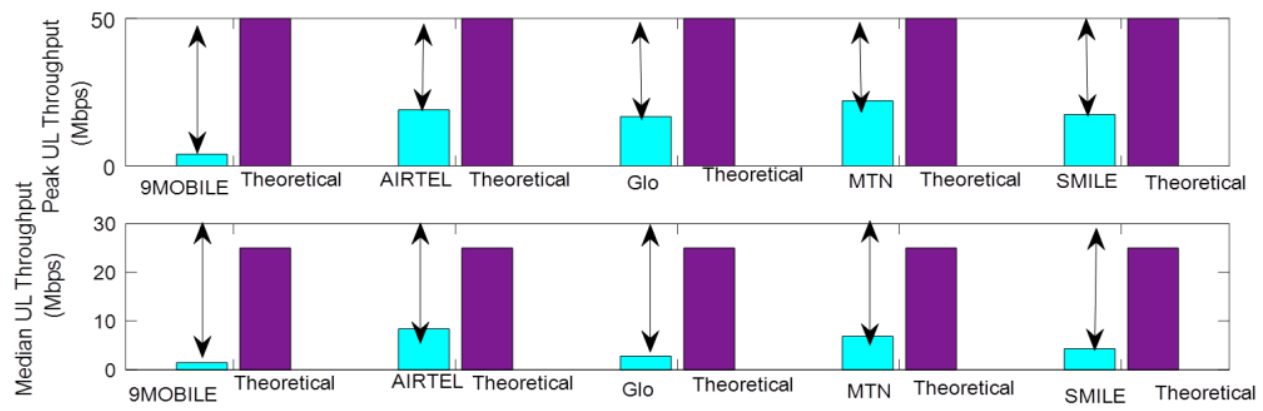

Figure 5. Peak and median physical layer UL throughput comparison among the Nigerian LTE MNOs

The result was not different from the DL counterpart. There seems to be pattern recognition in the DL and UL peak and median physical layer throughput among the Nigerian LTE MNOs. Based on the above analysis, it will be highly impossible for some of the networks to support multimedia broadcast multicast services (MBMS). With the introduction of shared DL and UL shared channel DL-SCH/UL-SCH configuration, the achievable UL and DL throughputs will further decrease in high traffic dense scenarios. RLC is a layer 2 protocol in LTE primarily for ARQ. The ARQ functionality provides error correction by retransmissions in acknowledged mode. Based on Figure 6, it could be seen the ULC throughput is slightly less than that of normal transmission. The reason for the decrease in throughput is attributed to the inclusion of overhead bytes, which subsequently lowers the transmitted payload. Indeed, the overhead will become prohibitively large in the face of poor RF channels. Latency is the duration between the transmission of the packet and the reception of acknowledgment. Low latency is the design goal of LTE networks. Two sources of network latency are the user plane and control plane latency. Based on the MIMO 4X4 configuration, LTE exhibits less than $100 \mathrm{~ms}$ latency from the control plane and less than $30 \mathrm{~ms}$ from the user plane. The control plane latency is the signaling required to switch the UE from the idle mode to the active mode involving the RRC connection and set up a dedicated mode.

User-Plane latency is defined as one-way transmit time between a packet that is available at the IP layer in the UE/E-UTRAN edge node and the availability of this packet at the IP layer in the E-UTRAN/UE node. User-Plane latency is vital for the performance of several applications. Many applications do not need a high data rate, but they do require low latency. Real-time gaming, voice, and interactive applications are some examples. Based on Figure 7, 9MOBILE possesses the worst round-trip delay among the Nigerian LTE MNOs with a value of over $1000 \mathrm{~ms}$. This is more than 10 percent in the ideal situation. This means that realtime gaming and interactive applications are not sustainable.

Int J Elec \& Comp Eng, Vol. 11, No. 3, June 2021 : 2191 - 2202 


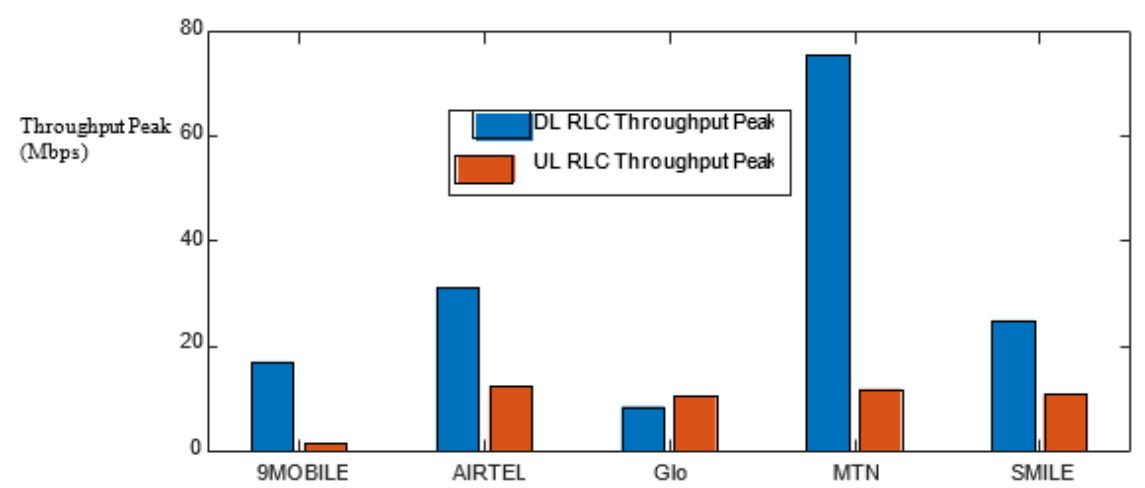

Figure 6. Comparing DL RLC and ULRLC throughput among the Nigerian LTE MNOs

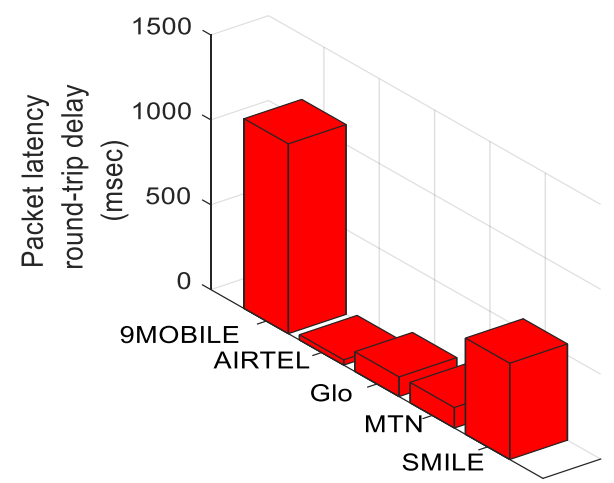

Figure 7. Packet latency round-trip delay among the Nigerian LTE MNOs

\subsection{Mobility test}

The 3GPP specifies that LTE will support low mobile speed $(0-15 \mathrm{~km} / \mathrm{h})$, but higher mobile speeds shall be supported as well as including high-speed train environments as a special case. A poor mobility performance will be visible in the high rate of handover failure rate and handover interruption time. The field results indicate that averagely Airtel has the highest S1/X2-based handover time of about $180 \mathrm{ms,} \mathrm{as} \mathrm{shown}$ in Figure 8, while 9MOBILE is attributed with lowest S1/X2-based handover time. The delay measured includes the air interface packet latencies needed to process the handover request sent by eNB and total processing time.

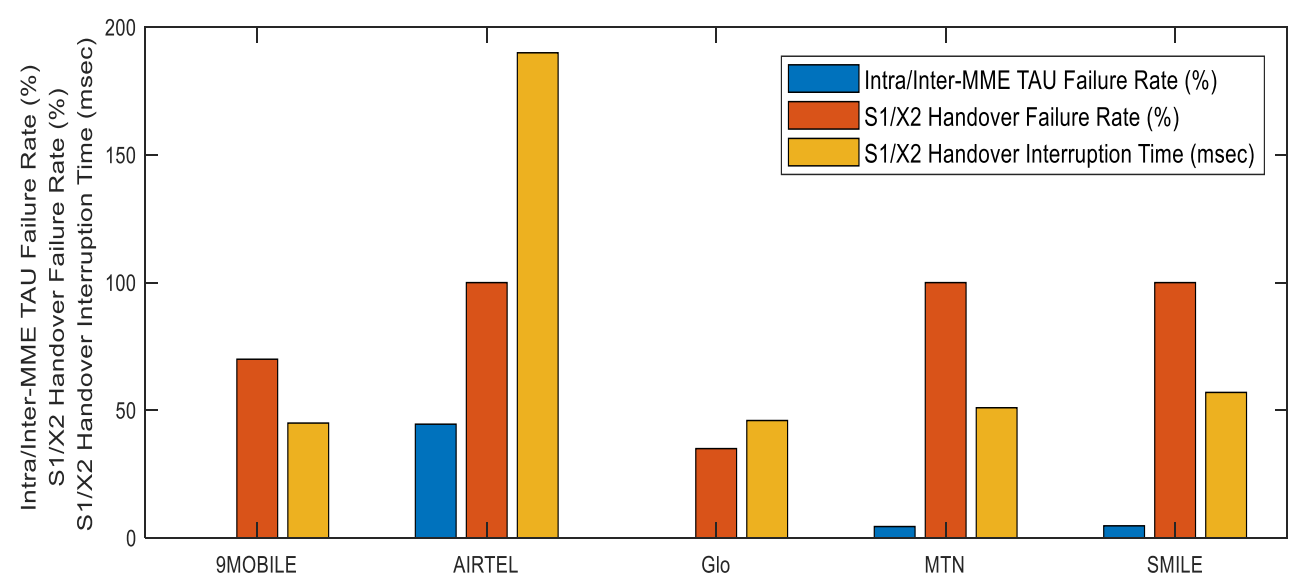

Figure 8. Mobility test performance among the Nigerian LTE MNOs 
Taken as for the S1/X2 handover failure rate, AIRTEL, MTN, and SMILE all show a close to 100 percent failure rate. Surprisingly, 9MOBILE has the least value of slightly above 60 percent. Apart from $\mathrm{S} 1 / \mathrm{X} 2$ handover failure rate and S1/X2 interruption time, tracking area (TA) and tracking area update plane (TAU) are another LTE mobility test metrics discussed. TA defines a collection of eNBs within a geographical area that makes up a coverage zone. TA comprises tracking area identity (TAI) and tracking area code (TAC). The unique global TAI is a function of the TAC and public land mobile network identifier (PLMN-ID). TAU is invoked when UE migrates from its registered TA, completes the attached procedure, and moves into the EPS mobility management (EMM)-registered state. Likewise, TAU is initiated by UE when it is in RRC Idle state or RRC Connected state, but the procedure ends only when the UE is in RRC connected state. As could be seen from Figure 8, the MNOs did not record any appreciable TAU mobility management entity (MME) failure. The mobility management entity is responsible for initial paging and authentication of mobile UE. Furthermore, MME is responsible for retaining the information about the location at the tracking area level for each user equipment as well as selecting the appropriate gateway during registration. Intra-MME TAU connotes a handover situation in which source and target eNBs are in the same TA while inter-MME TAU admits the handover between eNBs of different TAs.

\subsection{RF test}

The LTE RF test takes into account the following; RSRQ, SNR, SINR, reference signal received power (RSRP), receive signal strength indicator (RSSI). In this analysis, the focus is on SINR because it is a metric that can be easily verified and can be extended to derive others, as could be seen from (1)-(3). The SINR as a function of coverage area is a good indicator of the LTE physical layer QoS. SINR is an excellent candidate to grade the channel quality index (CQI). Channel quality index is a clear indication of the ability of LTE to support mobile file sharing, mobile audio, mobile web/data/VoIP. A high SINR is desirable for LTE as it will lead to lower HARQ and ARQ protocols and hence increases channel efficiency. This is contrary to the low SINR situation, which can only sustain mobile audio because the overhead is low. Based on Figure 9, all the MNOs exhibited strong and reliable coverage when the SINR was lowered to $-5 \mathrm{~dB}$. Meanwhile, when the SINR value was raised to $13 \mathrm{~dB}$, the area under decreased. In general, the physical layer QoS can be determined based on the block error rate (BLER). Channels with poor SINR values experience high BLER. In terms of modulation coding scheme (MCS), it means that MTN can support higher-order MCS higher-order modulation, based on reported SINR, the eNB scheduler selects the MCS assigned to the user. The 3GPP standard allows MCS indices of 0-31. The MCS range of 0-9 allows QPSK modulation, MCS 10-16 allows 16-QAM modulation, MCS 17-28 allows 64-QAM modulation usage, and the range of MCS 29-31 allows for special operation during retransmissions.

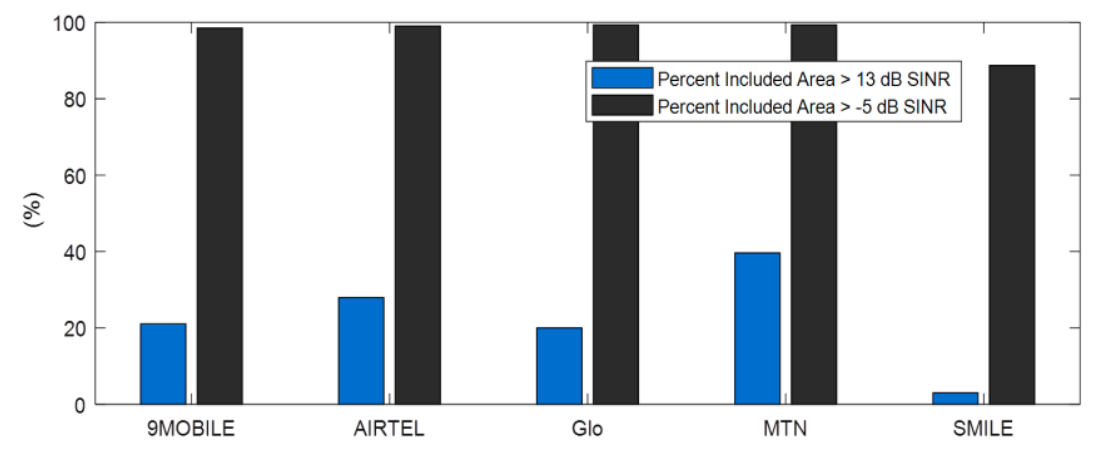

Figure 9. SINR test performance among the Nigerian LTE MNOs

\subsection{NIWBOR}

Right now, the networks in Nigeria are so weak that we have selected only metrics that clearly show the quality of RF deployment that has a direct impact on users. Many other KPIs can be used to measure different attributes. Most of these have significant relevance only after the networks have been adequately optimized.

The discussion on NIWBOR will be based on metrics already defined in (1). Figure 10 depicts the NIWBOR ranking using a pie-chart representation format. Based on the figure, 9MOBILE is attributed as having the highest percentage score of about 37\%. This simply implies that 9MOBILE has the highest deviation from the 3GPP LTE specifications. The MNO having the lowest score signifies having the optimal 
performance towards achieving the desired goals of the 3GPP standard based on release 8. Figure 11 shows various components that constitute the NIWBOR grading. As could be seen from Figure 11, all the Nigerian MNOs suffer from high latency. This implies that video streaming will be highly impossible to support. As it is now, mobile subscribers cannot enjoy the services of MBMS based on the latency issue. And at the same time, the MNOs cannot offer such services. Thus, losing some streams of revenue. LTE technology should bring to bear the advantages of enjoying fast file upload and download respectively. Therefore, it should facilitate the support of cloud-based gaming, immersive virtual reality services, real-time HD streaming, and conventional multimedia services. However, this is not the case in the Nigerian mobile data market. To keep high video quality the mobile network needs to provide high data rates instantaneously. Constant data rates are often preferred so that that video quality might suffer in high activity cases

\section{Ranking (LOWEST IS BEST)}

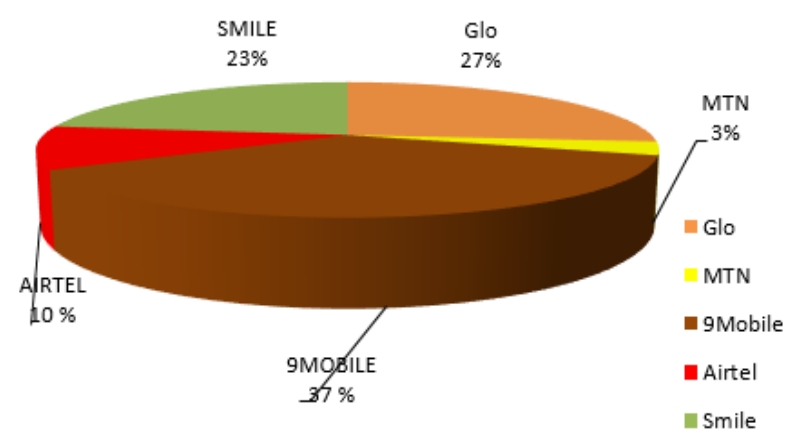

Figure 10. NIWBOR grading of Nigerian LTE MNOs in pie-chart representation

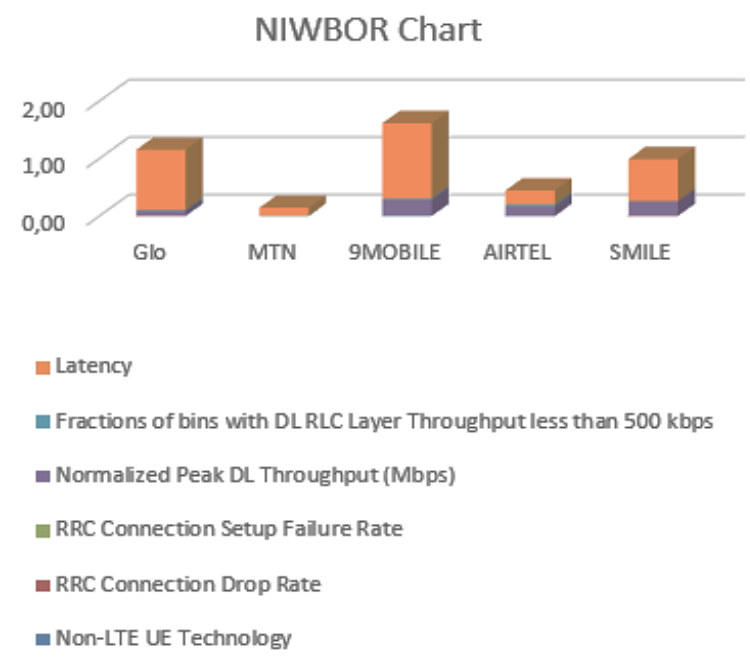

Figure 11. NIWBOR grading based on constituent components

\section{CONCLUSION}

In this work, a drive test was carried out to validate the real-world deployment of LTE networks in the Nigerian environment. It was discovered that all the Nigerian MNOs are performing below the 3GPPs recommended specification in-terms of uplink and downlink throughput, latency, RRC connection setup time, RRC connection drop rate, RRC connection setup failure rate. Though MTN has the best measured QoS, it still needs to enhance its service request delay time. Assertively, this will improve the time it takes for UEs to switch from idle mode to connected mode. Airtel needs to reduce the high S1/X2 handover interruption time. This will reduce the ping-pong effect of the UE's experience. Similarly, there is a need for most of the MNOs to improve on their physical layer DL throughput, as most of them are currently offering throughput, which is below 50 percent. The main limitation experienced in this work was that the mobility 
test was performed during the official working time, which is characterized by high vehicular traffic and high call traffic. The choice of the period was to fully understand the performance deterioration experienced by the users during working official time.

\section{ACKNOWLEDGEMENTS}

This work was supported by Covenant University and the authors acknowledge Enext wireless Inc. for providing the hardware and the software for this drive test exercise.

\section{REFERENCES}

[1] Nigerian Communications Commission (NCC), "Industry Statistics," 2018. [Online]. Available: https://www.ncc.gov.ng/stakeholder/statistics-reports/industry-overview.

[2] E. Agwu and A.-L. Carter, "Mobile phone banking in Nigeria: benefits, problems and prospects," International Journal of Business and Commerce, vol. 3, no. 6, pp. 50-70, 2018.

[3] H. U. Khan and A. C. Ejike, "An assessment of the impact of mobile banking on traditional banking in Nigeria," International Journal of Business Excellence, vol. 11, no. 4, pp. 446-463, 2017.

[4] O.O. Oni, A.A.A. Atayero, F.E. Idachaba, and A.S. Alatishe, "LTE Networks: Benchmarks, Prospects and Deployment Limitation," Proceedings of the World Congress on Enginerrin, 2014, pp. 422-427.

[5] R. Boateng, R. Hinson, R. Galadima, L. Olumide, "Preliminary insights into the influence of mobile phones in micro-trading activities of market women in Nigeria," Information Development, vol. 30, no. 1, pp. 32-50, 2014.

[6] E. Dahlman, S. Parkvall, and J. Skold, "4G: LTE/LTE-advanced for mobile broadband," Academic press, 2013.

[7] H. Zarrinkoub, "Understanding LTE with MATLAB: From Mathematical Modeling to Simulation and Prototyping," John Wiley \& Sons, 2014.

[8] Nigerian LTE service providers, 2018, [Online] Available: "http://www.enextwireless.com/network-providersdetails.

[9] A. M. Ghaleb, et al., "Throughput performance insights of LTE Release 8: Malaysia's perspective," 9th International Wireless Communications and Mobile Computing Conference (IWCMC), 2013, pp. 258-263.

[10] J. Huang, et al., "An in-depth study of LTE: effect of network protocol and application behavior on performance," Proceedings of the ACM SIGCOMM 2013 conference on SIGCOMM, vol. 43, no. 4, 2013, pp. 363-374.

[11] Nordin Rosdiadee, et al., "Empirical Study on Performance Evaluation Between Long Term Evolution (LTE), Third Generation (3G) and TV White Space Availability for Wireless Campus Network," International Journal of Simulation: Systems, Science and Technology, vol. 17, no. 32, pp. 25.1-25.9, 2016

[12] M. H. Alsharif, A. H. Kelechi, J. Kim, J. H. Kim, "Energy efficiency and coverage trade-off in 5G for eco-friendly and sustainable cellular networks," Symmetry, vol. 11, no. 3, p. 408, 2019.

[13] A.P. Iyer, I. Stoica, M. Chowdhury, and L.E. Li, "Fast and Accurate Performance Analysis of LTE Radio Access Networks," arXiv preprint arXiv:1605.04652, 2016.

[14] J. Huang, et al., "A close examination of performance and power characteristics of 4G LTE networks," MobiSys '12: Proc. of the 10th international conference on Mobile systems, applications, and services, 2012, pp. 225-238.

[15] M. Z. Shafiq, et al., "Understanding the impact of network dynamics on mobile video user engagement," The 2014 ACM international conference on Measurement and modeling of computer systems, 2014, pp. 367-379.

[16] A. Alcatel-Lucent, "9959 network performance optimizer," 2014, [Online] Available: http://www.alcatellucent.com/products/ 9959-network-performance-optimizer.

[17] E. ERICSSON, "Ericsson RAN analyzer," 2014. [Online] Available: http://www.ericsson.com/ourportfolio/products/ran-analyzer.

[18] G. RP-170779, "Study on enhanced LTE support for aerial vehicles, NTT DOCOMO, Ericsson," 2017 [Online]. Available: http://www.3gpp.org/ftp/tsg_ran/tsg_ran/TSGR_75/Docs/RP-170779.zip."

[19] G. T. 36.777, "Enhanced LTE support for aerial vehicles," 2018, [Online] Available: ftp://www.3gpp.org/specs/archive/36_series/36.777.

[20] S. Chen, and C. Zhu, "ICI and ISI analysis and mitigation for OFDM systems with insufficient cyclic prefix in time-varying channels," IEEE Transactions on Consumer Electronics, vol. 50, no. 1, pp. 78-83, 2004

[21] R. Stirling-Gallacher Richard, et al., "Multi-user and single user MIMO for communication systems using hybrid beam forming," U.S. Patent No. 9,008,222. Washington, DC: U.S. Patent and Trademark Office, 2015.

[22] T. O. Takpor and F. E. Idachaba, "Analysis and Simulation of LTE Downlink and Uplink Transceiver," Proceedings of the World Congress on Enginerring, vol. 1, 2014, pp. 2-7.

[23] O. E. Agboje, S. O. Adedoyin, and C. U. Ndujiuba, "State of Fiber Optic Networks for Internet Broadband Penetration in Nigeria-A Review," International Journal of Optoelectronic Engineering, vol. 7, no. 1, pp. 1-12, 2017.

[24] N. C. Commission, "2014 Year End Subscriber/Network Data Report for Telecommunication Operating Companies in Nigeria," Policy Competition and Economic Analysis Department, pp. 1-23, 2014.

[25] 3GPP, "3rd Generation Partnership Project; Technical specification group radio access network; Evolved universal terrestrial radio access (E-UTRA); User equipment (UE) radio transmission and reception," Technical Specification, pp. 1-169, 2011. 